\title{
The Application of Layering Technology in Computer Software Development
}

\author{
Luo Qiqiang ${ }^{1}$, Chen Shuang ${ }^{2}$ \\ ${ }^{1}$ Nanchang Institute of Science and Technology, Nanchang, China \\ ${ }^{2}$ Jiangxi Tellhow Animation Vocational College, Nanchang, China
}

Keywords: layering technology; computer software development; research on application

\begin{abstract}
At present, with the accelerating development of Chinese science and technology, the development of computer information technology has entered in a new stage, bringing great convenience for people's daily life and work. In the era, the development of computer software technology gets deeper and layering technology occupies an important position in software development engineering. Layer-management of computer software can improve the efficiency of computer software in operation to a great extent, so that people can realize the significance of layering technology in computer software development. The application of layering technology has become the main trend for the industry of computer software development currently. The development of computer software technology is an important signal of China's scientific and technological development. The application of layering technology enhances the satisfaction of users, which also indicates the advent of a new era, and the transformation of layering technology means the in-depth development of the industry. Therefore, this paper explores and analyzes the application of layering technology in computer software development, and provide personal opinions, so as to provide reference for deeper research of layering technology.
\end{abstract}

During the recent decade, the development of Chinese computer software is growing, and more and more innovative achievements of software have been applied to people's work and life and obtain ideal effects. At present, Chinese computer technology has entered a brand new development stage. With the extensive application of computer technology in all walks of life, people's recognition of computer technology becomes more comprehensive, and the prosperous computer technology has met people's demand of life and work to a great extent [1]. With the intensifying and improving technological means and technological diversification, people's expectation for computer technology becomes higher, thus simple computer software development cannot satisfy users' needs, which requires innovations on traditional computer software development. The overall development of Chinese science and technology promotes the development of computer software engineering, with the original two-layer structure changing to three-layer structure, layer network becoming clearer, and the overall operation efficiency being improved. To apply layering technology to computer software development can provide high-quality services for users.

\section{Introduction to Basic Concept and Characteristics of Software Layering Technology}

\subsection{The basic concept of software layering technology}

The software layering technology, due to the certain dependencies between computer software structures, mainly refers to the management of software, so that the overall operating efficiency and accuracy of the software can be effectively improved. Flexibility is one of the most critical features in the operation of computer software, which can effectively improve the quality of software operation. The computer software development is not a single study of technical characteristics, but to fully grasp the details of the operation of computer software, so that the functions of computer software become more diverse. In the early 1980s, the single-layer computer software was in development. The application of small data in computer software is fruitful, which can effectively improve the overall operating efficiency of computer software. In computer software development, people constantly realize the importance of computer software development and the significance of 
multi-layer network structure construction in computer software. In short, the application of layering technology in computer software development, breaks through the processing mode of single-business of traditional computer software and creates multi-layer technology, so that it enters a brand new stage [2].

\subsection{The characteristics of software layering technology}

The software layer technology, due to its unique advantages, is appropriately applied in computer software development to ensure the overall operating quality and improve its operating efficiency. Layering technology, conductive to the application and innovation of computer software, can disintegrate the complex system in computer software development according to its functional differences, so as to further improve the overall effects of computer software development. The application of layering technology can effectively correct a certain functional layer inside computer system, to enhance the quality of software development [3]. At the same time, its application can shorten the time for computer software development, conductive to the re-application of computer software. The adoption of standard port can effectively realize the seamless connection of port, which is also a technological innovation.

In computer software development, it is necessary to improve and innovate the original functions of computer software, so as to enhance the development quality of software products. Layering technology can accurately and reasonably allocate the inside and outside relationship in computer software development. By virtue of many advantages, compared to traditional multi-layer network structure, it can greatly promote the work efficiency of computer software developers and extend application cycle of software.

\section{The Specific Applications of Layering Technology in Computer Software Development}

In developing computer software the application of layering technology greatly promotes the innovations of software development. Nowadays, the accelerating social and economic development in China and a new stage of scientific and technological development provide beneficial environment for the development of computer technology in China. The development of computer software breaks through traditional single-business processing mode and begins continuously multi-layer computer software development processing, changing from double-layer to three-layer and four-layer structure. The applications of layering technology in computer software development are presented [4].

\subsection{The practical application of double-layer technology in computer software development}

In developing computer software, the application of double-layer technology is conductive to improving the overall efficiency of software development. Besides, it can shorten the period for software development to a great extent. The constituents between client-side and servers mainly rely on double-layer network technology, which brings new possibilities for the processing of network logical relations and processes network technology problems in a timely manner. Generally, the main function of server is to provide important information required by users, so that clients can know the changes of relevant true information at the first time. For computer servers without good efficacy, the double-layer technology cannot be applied in software development. Thus, to ensure the overall performance of computer is the primary premise for software development [5]. In addition, the number of computer software users cannot be too large, and shall be controlled within a certain range according to the standard. Once the amount of users dramatically increases, there will be errors in the operation of system, so that the operation will be slower. In this way, the actual requirements of users cannot be well satisfied.

\subsection{The application of three-layer technology in computer software development}

In the development of computer software, the application of three-layer technology is very important, which can effectively improve the overall operating efficiency of the software. Compared with traditional technology, the three-layer technology mainly increases the number of 
application servers, and also makes it easier to store user data [6]. In the process of computer software development, the application of three-layer software technology can effectively improve the information access efficiency and access accuracy of the computer, and better realize the information exchange between the computer and people, thus making the information conversion become faster.

In general, the three-layer technology mainly includes three levels, namely, the processing of business, the processing of the computer interface, and the processing of relevant important data. First of all, the processing of business mainly refers to the accurate analysis of users' information, so as to truly grasp the actual requirements of users. Starting from the starting point of the request can effectively improve the efficiency of sorting related data [7]. Secondly, the interface processing mainly refers to the active exploration of users' needs. In the process of exploration, attention should be paid to the precise processing of useful data information, so as to realize the scientific analysis of the information, and finally report the processed results to the target service users in time. The comprehensive application of three-layer technology in computer software development effectively improves the overall working efficiency of computer software. Under the current user environment, the application of three-layer technology is the inevitable trend of the industry development, promoting the overall development of the computer software engineering [8].

\subsection{The application of four-layer technology in computer software development}

At present, the development speed of China's Internet information technology is accelerating, and the operating environment of computer software is becoming more diversified, which puts higher requirements on the operation quality and operational efficiency of computer software, and the challenges faced by the development of the industry are severer. As the complexity of data sources has increased to a large extent, data processing requirements have become more complex. At this stage, Chinese users have also put forward more personalized and diversified requirements for the actual functions of computer software. Under such circumstances, relevant technical personnel should deepen their research based on the double-layer technology and three-layer technology so as to meet modern users' actual needs. Due to the increasingly diverse environment in which computer technology is located in China, the deficiencies in double-layer technology and three-layer technology are increasingly exposed, which limits the diversity and complexity of users to a certain extent. At this time, four-layer technology came into being. The main advantage is that it effectively reduces the interaction between the various levels, and also enhances the independence between the layers, so that the interference from external factors for computer software in the process of operation is greatly reduced [9]. In terms of data processing, the accuracy has been greatly improved, and data is exchanged between them, thereby improving the overall efficiency of software development.

\subsection{The application of middleware technology in computer software development}

At present, China's social and economic development speed is accelerating, and people's overall living standards have also been greatly improved. The development of social economy has greatly promoted the development of computer software technology. When developing computer software technology, middleware technology is a relatively independent system software compared to other technology types. The middleware can effectively shield the complex technology that is mixed in the distribution set. In the process of developing computer software technology, it is necessary to pay attention to effectively reduce the difficulty of the development technology, so that the cycle for the application of middleware technology in the software system can be effectively shortened [10]. In addition, the effective application of middleware technology can reduce the risks of software operation, ensure the secure operation of computer system, and give full play to more advantages of computer software system, so that negative factors will be overcome and advantages of system resources can be better reflected. 


\section{The Practice of Multi-layer Technology in Computer Software Development}

At present, the speed of China's social and economic development is accelerating. Under the background of this era, the development of many industries has ushered in a new spring. The development pace of China's catering and department stores is constantly increasing, and its scale is constantly expanding, and shop information building has become a key task in development. Layering technology has strong adaptability. In teamwork, it is required to pay attention to the accuracy of layering management, which is crucial, and only in this way can it better satisfy the higher requirements for software development. In the process of computer software store management, it is necessary to make efforts to realize the information construction of shops. In the process of managing the shops, the main functions of the system operation include system, operation, administration, marketing, etc., and the follow-up management. In terms of the store management business, the scope is relatively wide, involving multiple steps, and there are differences in different management standards for shops. Therefore, when managing shops, it is necessary to pay attention to the effective application of layering technology, which can ensure the quality of store management to a great extent.

\section{Conclusion}

The development of computer technology in China reaches a new height, which is directly related to the extensive application of computer technology. The computer software brings convenience for users' work and life. In developing software, it is necessary to stress the extensive application of layering technology. Nowadays, the development of layering technology in China is still faced with many challenges. With the improving science and technology in China, people's requirements for computer software development become higher. It is difficult for traditional double-layer technology to meet the requirements of the times currently. Therefore, it is necessary to conduct innovations to relate it closed to the industrial development. The security and information accuracy of multi-layer technology is stronger, which brings better operation experience for users and greatly enhances the overall information processing ability so as to realize ideal effects in practical application. In addition, the software technology with multi-layer structure shortens the period for software development to a great extent. The multi-layer technology plays an important role in software development, which is conductive to the development of computer software technology in China and the overall improvement of Chinese science and technology. In a word, with the complex computer environment, computer software development enters a brand new stage, and relevant technicians in the industry shall recognize their important responsibilities so as to promote the overall development of computer software in China.

\section{References}

[1] Huang Jinren. Research on the Application of Layering Technology in Computer Software Development [J]. Digitalization User, 2018,24(49):122.

[2] Ma Di, Hu Huijuan. Strategies of the Application of Layering Technology in Computer Software Development [J]. Science and Technology Innovation, 2018,22(31):84-85.

[3] Liu Yu. The Application of Layering Technology in Computer Software Development [J]. Heilongjiang Science, 2018,9(20):86-87.

[4] Dai Chenyu. The Application of Layering Technology in Computer Software Development [J]. Science and Technology \& Innovation, 2018,3(20):148-149.

[5] Cao Lingling. On the Application of Layering Technology in Computer Software Development [J]. Digital Communication World, 2018,20(10):269.

[6] Jiang Qingchao. On the Application of Layering Technology in Computer Software Development [J]. Juanzong, 2018,5(26):225.

[7] Lu Zhaoxian. On the Application of Layering Technology in Computer Software Development [J]. Information System Engineering, 2018,11(9):91. 
[8] Pu Tianhong. The Application of Layering Technology in Computer Software Development [J]. Computer Knowledge and Technology, 2018,14(25):55-56.

[9] Wu Xi. The Application of Layering Technology in Computer Software Development [J]. Digitalization User, 2018,24(35):160.

[10] Wang Yayun. Research on the Application of Layering Technology in Computer Software Development [J]. Peak Data Science, 2018,28(7):8. 